\title{
Mariposas (Lepidoptera: Papilionoidea) de Tierradentro, San Andrés de Pisimbalá, Cauca, Colombia
}

\author{
Butterflies (Lepidoptera: Papilionoidea) from Tierradentro, San Andrés de Pisimbalá, Cauca, \\ Colombia
}

\section{Germán Darío Velasco-Rojas ${ }^{1 *}$, Karen Nathalia Gallego-Cotazo ${ }^{1}$, Jorge Mario Becoche-Mosquera ${ }^{1}$, Ivon Andrea Bolaños-Martínez ${ }^{2}$}

\begin{abstract}
Resumen
Este trabajo es el primer registro sobre la caracterización de la diversidad de lepidópteros (Papilionoidea) en el Parque Arqueológico de Tierradentro, vereda de San Andrés de Pisimbalá, Inzá, Cauca, Colombia, considerada como una región ecoturística y de importancia cultural para el país. Se establecieron recorridos a lo largo de tres coberturas vegetales identificadas como bosque secundario, cultivos de pancoger y potrero. Para la recolecta de mariposas se emplearon dos métodos de muestreo, el primero con red entomológica y el segundo mediante el uso de trampas tipo Van Someren Rydon. Se registró un total de 128 especies, distribuidas en 99 géneros y cinco familias (Nymphalidae, Hesperiidae, Pieridae, Riodinidae y Lycaenidae). Las coberturas de bosque y cultivos presentaron la mayor diversidad, probablemente por la heterogeneidad vegetal observada. Adicionalmente, se encontró que el bosque presentaba algunas especies indicadoras que se asocian con buenos estados de conservación.
\end{abstract}

Palabras clave: diversidad, papilionoideos, coberturas vegetales, San Andrés de Pisimbalá

\begin{abstract}
This work is the first report on the characterization of Lepidoptera diversity (Papilionoidea) in Tierradentro Archaeological Park, San Andrés de Pisimbalá, Inzá, Cauca, Colombia, considered an ecotourism region of cultural importance for the country. Routes were established along secondary forest, pancoger crops and pastures. To collect Lepidoptera, two sampling methods were used, the first using an entomological net and the second using van Someren Rydon traps. A total of 128 species distributed in 99 genera and five families (Nymphalidae, Hesperiidae, Pieridae, Riodinidae and Lycaenidae) were collected. Forest cover and pancoger crops were the most diverse, probably due to the plant heterogeneity observed. Additionally, it was found that the forest presented some indicator species that are associated with good conservation states.
\end{abstract}

Keywords: Diversity, papillionoids, plant covers, San Andrés de Pisimbalá

\footnotetext{
1. Grupo de Estudios en Geología, Ecología y Conservación - GECO, Universidad del Cauca.

2. Grupo Biología Molecular Ambiental y Cáncer - BIMAC, Universidad del Cauca. Grupo Ciencias Básicas y Clínicas de la Salud - Pontificia Universidad Javeriana Cali.
}

* Autor para correspondencia: <germanvel@unicauca.edu.co> 


\section{INTRODUCCIÓN}

La diversidad biológica y el estudio de la ecología de las poblaciones han sido un tema central para promover y ejecutar objetos de conservación del entorno natural (MADS y PNUD, 2014). Colombia, como país megadiverso, ocupa los primeros puestos en cuanto a diversidad de especies (Andrade, 2011). En el caso de las mariposas, Colombia posee un total de 3276 especies, dentro de las cuales 350 son endémicas; la riqueza de estos organismos ubica al país como el segundo con mayor diversidad después de Perú (Henao y Gantiva, 2020).

Lepidoptera constituye el tercer orden de insectos con más especies descritas después de Coleoptera y Diptera, contribuyendo a la dinámica ecosistémica al ser partícipes de procesos mutualistas como la polinización o formando parte de la cadena trófica (Villalobos y Salazar, 2020). Además, las mariposas son usadas como bioindicadores del estado de conservación del entorno natural a causa de su sensibilidad a los cambios ambientales (contaminación de fuentes hídricas, cambio climático, extensión de la frontera agropecuaria e implementación de cultivos de uso ilícito) que generan la destrucción del hábitat, los cambios en la cobertura, el uso del suelo y la desaparición o desplazamiento de especies (Andrade, 1998; Morales, 2011; Sánchez, 2002; Tacuma, 2020), por lo que la abundancia y diversidad de algunas especies de estos insectos deja al descubierto la salud de un entorno debido a que estos factores están directamente relacionados con su estado de conservación.

Adicionalmente, la aceleración de estos procesos antrópicos en regiones apartadas o inexploradas por sus condiciones de difícil acceso o de conflicto, hace prioritaria la necesidad de conocer la diversidad de flora y fauna con el fin de proponer en esos territorios, alternativas de restauración ecológica a mediano y largo plazo. Lo anterior debido al aumento de la deforestación en algunas áreas de Colombia después del posconflicto, condición que puede afectar la conservación de los organismos objeto de esta investigación (Clerici et al., 2020).

Por lo antes expuesto, este estudio tiene por objetivo conocer la diversidad de papilionoideos en la vereda de San Andrés de Pisimbalá, municipio de Inzá, departamento del Cauca y así facilitar la promoción de acciones que contribuyan a su sostenibilidad, teniendo en cuenta el potencial ambiental, turístico y económico que se proyecta en la región. Además, este estudio se convertirá en una herramienta adicional con la que se busca promover el turismo sostenible en la región.

\section{MATERIALES Y MÉTODOS}

\section{Área de estudio}

La vereda de San Andrés de Pisimbalá $\left(02^{\circ} 34^{\prime} 57^{\prime \prime} \mathrm{N}\right.$ y $76^{\circ} 02^{\prime} 42^{\prime \prime} \mathrm{W}$ ) está ubicado en el municipio de Inzá, a una distancia de $110 \mathrm{~km}$ de la ciudad de Popayán, sobre la vertiente oriental de la cordillera Central, a una altitud de $1702 \mathrm{~m} \mathrm{s.} \mathrm{n.} \mathrm{m.} \mathrm{La} \mathrm{topografía} \mathrm{es}$ variada, resultado de un relieve heterogéneo con una geomorfología de montañas ramificadas casi verticales con crestas agudas y de laderas cortas (Pachón et al., 1996). Sus suelos son húmedos por causa de la presencia de afloramientos hídricos y de acuerdo con Holdridge (1967) es una región con vegetación tropical, por lo que se considera una transición de bosque húmedo a muy húmedo/montano bajo (Bmh$\mathrm{Bh} / \mathrm{MB}$ ) (figura 1).

\section{Recolección del material biológico}

Se llevaron a cabo seis salidas de campo durante los meses de enero a junio del año 2019, con una duración de cuatro días por muestreo. Se establecieron cuatro transectos de $200 \mathrm{~m}$ a lo largo de diferentes hábitats: Transecto 1: Potrero, Transecto 2-4: Cultivos de pancoger y Transecto 3: Bosque secundario, donde se emplearon muestreos con red entomológica y trampas tipo van Someren Rydon (Andrade et al., 2013; Villarreal et al., 2006).

\section{Muestreo con red entomológica}

Se realizaron muestreos libres, en un horario de 8:00 a 11:00 y de 15:30 a 18:00 (esfuerzo de muestreo por persona, un total de $6 \mathrm{~h}$ de observación y captura por día. Este muestreo lo realizaron tres personas) para completar el registro de especies que presentan hábito crepuscular (Pollard y Yates, 1996). Se pro- 


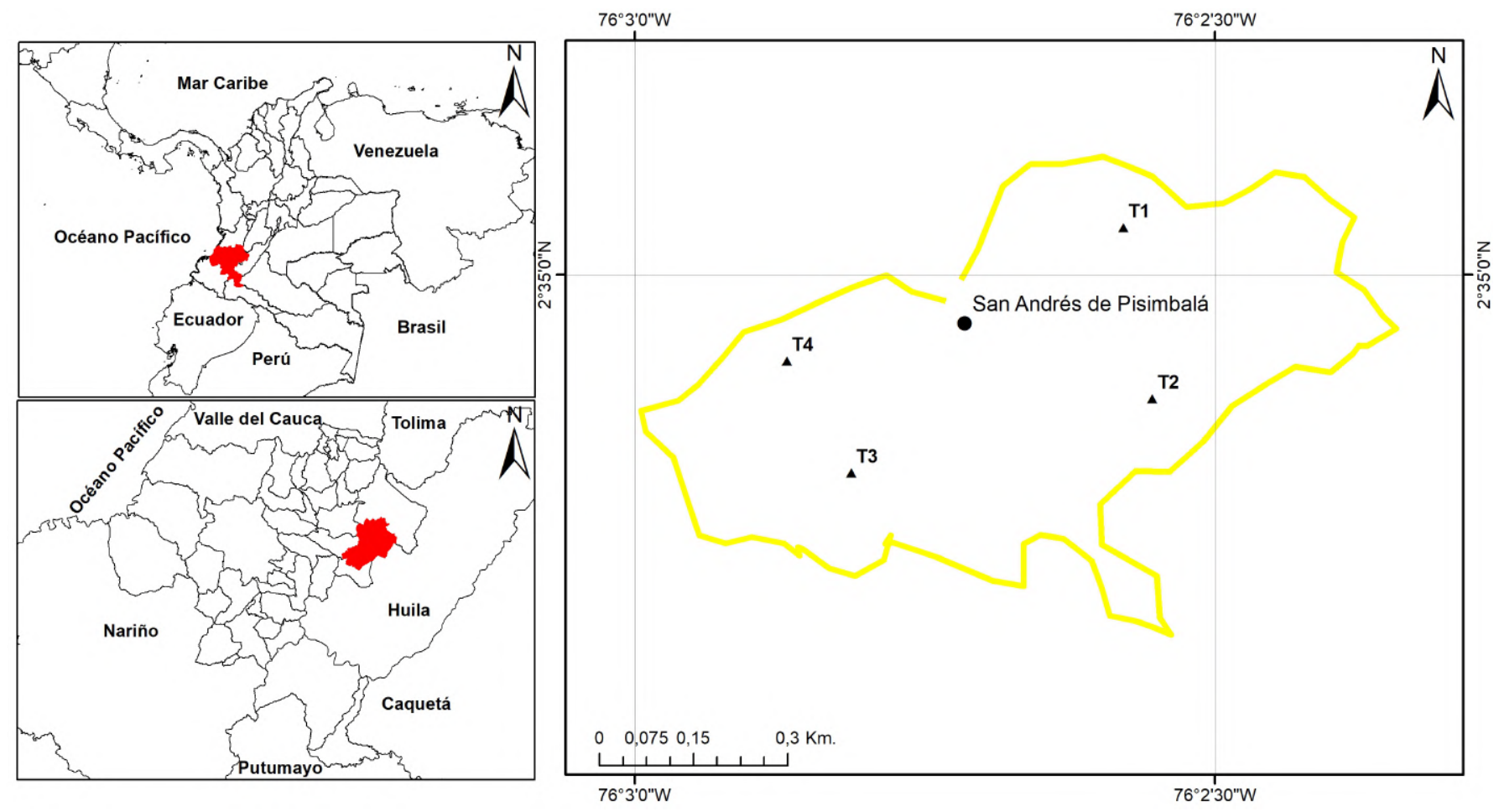

Figura 1. Localización geográfica de la vereda de San Andrés de Pisimbalá (Inzá, Cauca).

curó siempre que el esfuerzo de muestreo fuera con el mismo horario y personal. Se registró el tiempo muestreado en cada cobertura y la hora en que se recorrió.

\section{Muestreo con trampas tipo Van Someren Rydon}

Se instalaron diez trampas dentro de cada una de las coberturas identificadas; como cebo se utilizó fruta fermentada (banano y piña). Se realizaron transectos de $200 \mathrm{~m}$ en cada cobertura, donde se instalaron diez trampas separadas entre sí cada $20 \mathrm{~m}$. Las trampas fueron colgadas entre uno y tres metros por encima del suelo, dependiendo de la estructura de la vegetación y altura del dosel. Las trampas se activaron por espacio de 48 h, revisándose cada tres horas (Daily y Ehrlich, 1995) con un esfuerzo de muestreo por transecto de $480 \mathrm{~h}$.

Todos los ejemplares capturados fueron sacrificados por medio de cámara letal, usando acetato de etilo, con el propósito de no deteriorar el individuo. Las mariposas se guardaron en sobres de papel mantequilla con sus respectivos datos (localidad, departamen- to, municipio, vereda, lugar, fecha de recolecta, hora, nombre del colector, altitud y coordenadas geográficas tomadas con un GPS directamente en campo) para su posterior montaje y análisis (Andrade et al., 2013).

\section{Identificación taxonómica del material}

Se utilizaron los patrones de coloración, forma alar, las bandas y algunas estructuras especiales como palpos, patas y órganos genitales como caracteres taxonómicos definidos para la determinación de los ejemplares colectados (Andrade et al., 2013).

En cuanto a la determinación taxonómica, se utilizaron las claves, ilustraciones y descripciones de Andrade (1990, 1995, 2002), Constantino (1995), D'Abrera (1984, 1987a, 1987b, 1994, 1995), García et al. (2002), Le Crom et al. (2002), Le Cromet al. (2004). Además, se siguió la clasificación taxonómica de Lamas (2004) propuesta en Butterflies of America (Warren et al., 2017). La recolección del material biológico se realizó bajo la Resolución 0152 del 12 de febrero de 2015 emitida por la Autoridad Nacional de 
Licencias Ambientales - ANLA por el cual se otorgó un permiso marco de recolección de especies silvestres de la diversidad biológica con fines de investigación científica no comercial. El material debidamente montado y etiquetado se depositó en la colección entomológica del Museo de Historia Natural de la Universidad del Cauca (MHN-UCC) en la ciudad de Popayán.

\section{Análisis de datos}

En primer lugar, se elaboró el listado de las especies encontradas en la zona de estudio; también se realizaron gráficos para representar las familias, géneros y especies más dominantes. Para el análisis de la eficiencia del muestreo, se construyó la curva de interpolación y extrapolación basada en la cobertura de la muestra, que es la proporción del total de individuos en una comunidad representada por las especies observadas (Chao y Jost, 2012). Además, se determinó la diversidad verdadera por medio de los números efectivos de especies (Jost, 2006). Se calculó la diversidad de orden cero $\left({ }^{0} \mathrm{q}\right)$ que equivale a la riqueza de especies, orden uno $\left({ }^{1} \mathrm{q}\right)$ equivalente a la frecuencia de especies sin tener en cuenta si son comunes o raras (exponencial de Shannon) y orden dos $\left({ }^{2} \mathrm{q}\right)$ que representa las especies dominantes (inverso de Simpson) siguiendo el método propuesto por Jost (2006) y Chao y Jost (2015).

Todos los análisis se realizaron por medio del software R Studio versión 1.1.463 (R Core Team, 2021). La diversidad verdadera y la curva de interpolación y extrapolación basada en la cobertura de la muestra se calcularon con los paquetes estadísticos iNEXT (Hsieh et al., 2013) y SpadeR (Chao et al., 2015) y paquetes complementarios como knitr (Xie, 2014) ggplot2 y dplyr (Wickham, 2016). Por último, se calculó el índice de similitud de Bray-Curtis con los paquetes vegan (Oksanen et al., 2020) y stats (R Core Team, 2021).

\section{RESULTADOS}

Se registró un total de 128 especies distribuidas en 99 géneros, cinco familias (Nymphalidae, Hesperiidae, Pieridae, Riodinidae y Lycaenidae) y 551 individuos (Anexo 1), representando el $4 \%$ de las especies regis- tradas para Colombia por Andrade (2011).

Basado en el método de interpolación/extrapolación, la cobertura de la muestra para los tipos de coberturas vegetal estudiadas arrojó valores que oscilan entre el $48 \%$ y $85 \%$, revelando que es posible encontrar más especies de mariposas según aumente el esfuerzo de muestreo (tabla 1, figura 2). Entonces, el bosque secundario registra una cobertura de muestra del $85 \%$, seguido de cultivos de pancoger $(80 \%)$ y potreros $(48 \%)$.

Tabla 1. Integridad del muestreo

\begin{tabular}{l|c|c|c}
\hline Tipo de cobertura & $\mathbf{n}$ & S. obs & SC \\
\hline Bosque secundario & 333 & 106 & 0,85 \\
Cultivos de & 178 & 67 & 0,8 \\
pancoger & 40 & 29 & 0,48 \\
\hline Potrero & \multicolumn{2}{|l}{} \\
\hline
\end{tabular}

n: abundancia, S. obs: especies observadas, SC: cobertura de la muestra.

La familia Nymphalidae predominó con mayor riqueza y abundancia de especies (54/363) lo que representa un $42 \%$ del total registradas, seguida de Hesperiidae (40/77), Pieridae (16/66), Lycaenidae $(12 / 23)$ y por último Riodinidae $(6 / 22)$ (figura 3$)$.

Las especies más dominantes fueron Anartia amathea (Linnaeus, 1758) con 41 individuos, Oressinoma typhla E. Doubleday, [1849] (28), Heliconius cydno (E. Doubleday, 1847) (26), Heliconius clysonymus Latreille, [1817] (24), Euptychoides saturnus (A. Butler, 1867), Siproeta epaphus (Latreille, [1813]) (21) y Actinote anteas (E. Doubleday, [1847]) con 20 registros (figura 4).

La cobertura vegetal con la mayor representatividad de especies fue el bosque secundario donde se registraron 106 especies y 333 individuos, seguida de los cultivos de pancoger con 67 especies y 178 individuos y potrero con 29 especies y 40 individuos (figura 5).

En cuanto a la diversidad verdadera se encontró que en el bosque secundario el orden ${ }^{0} \mathrm{q}$ (riqueza de especies) fue mayor respecto a las demás coberturas; intermedia en cultivos de pancoger y baja en potrero. 


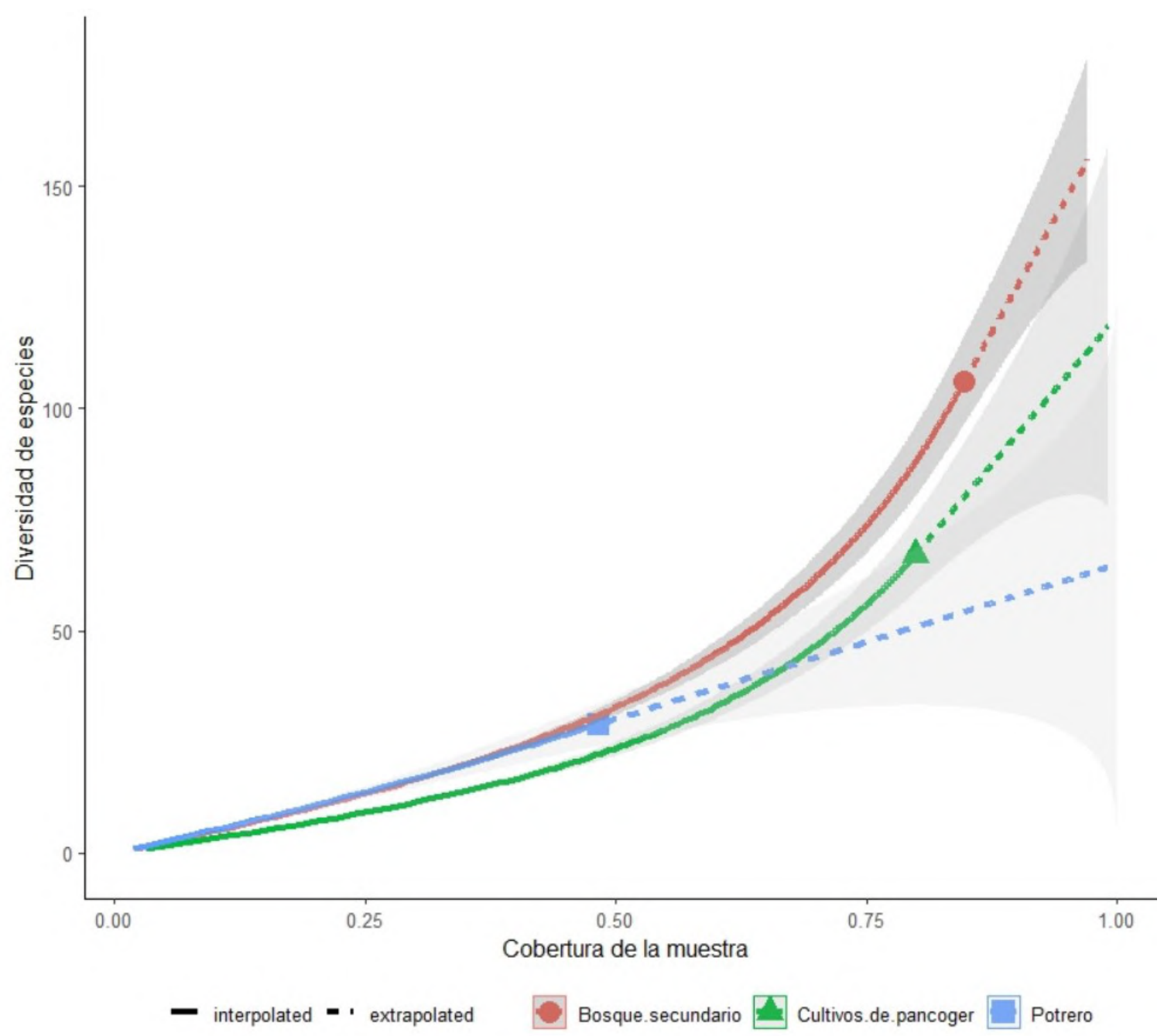

Figura 2. Curva de interpolación y extrapolación basada en la cobertura de la muestra.

De igual manera se registran en ese mismo orden de coberturas un número de especies frecuentes respecto al orden de diversidad ${ }^{1} \mathrm{q}$. Sin embargo, con la medida de diversidad ${ }^{2} \mathrm{q}$ se encontró que las coberturas de cultivos de pancoger y potreros son similares debido a que presentan un número semejante de especies dominantes (tabla 2, figura 6).

El índice de Bray-Curtis indica que se conformaron tres grupos teniendo en cuenta los tipos de cobertura. El primero está conformado entre bosque y cultivos con una similitud del 52,8\%, donde se comparten 36 especies, siendo las más comunes Caligo oileus C. Felder y R. Felder, 1861, Tegosa anieta (Hewitson, 1864), H. clysonimus, O. typhla y S. epaphus. Por último, el grupo hermano adyacente al anterior conjunto es el potrero con una similitud del $16 \%$, en cuanto a la estructura de papilionoideos, respecto a las demás coberturas (figura 7).

\section{DISCUSIÓN}

La efectividad de la captura de mariposas para las coberturas de bosque secundario y cultivos de pancoger estuvo bien representada, con más del $80 \%$ de la comunidad, siendo el potrero donde se registró el menor número de especies, esto posiblemente a las condiciones climáticas adversas (precipitaciones altas) que afectaron el registro de especies en estas zonas $(<48 \%)$. 


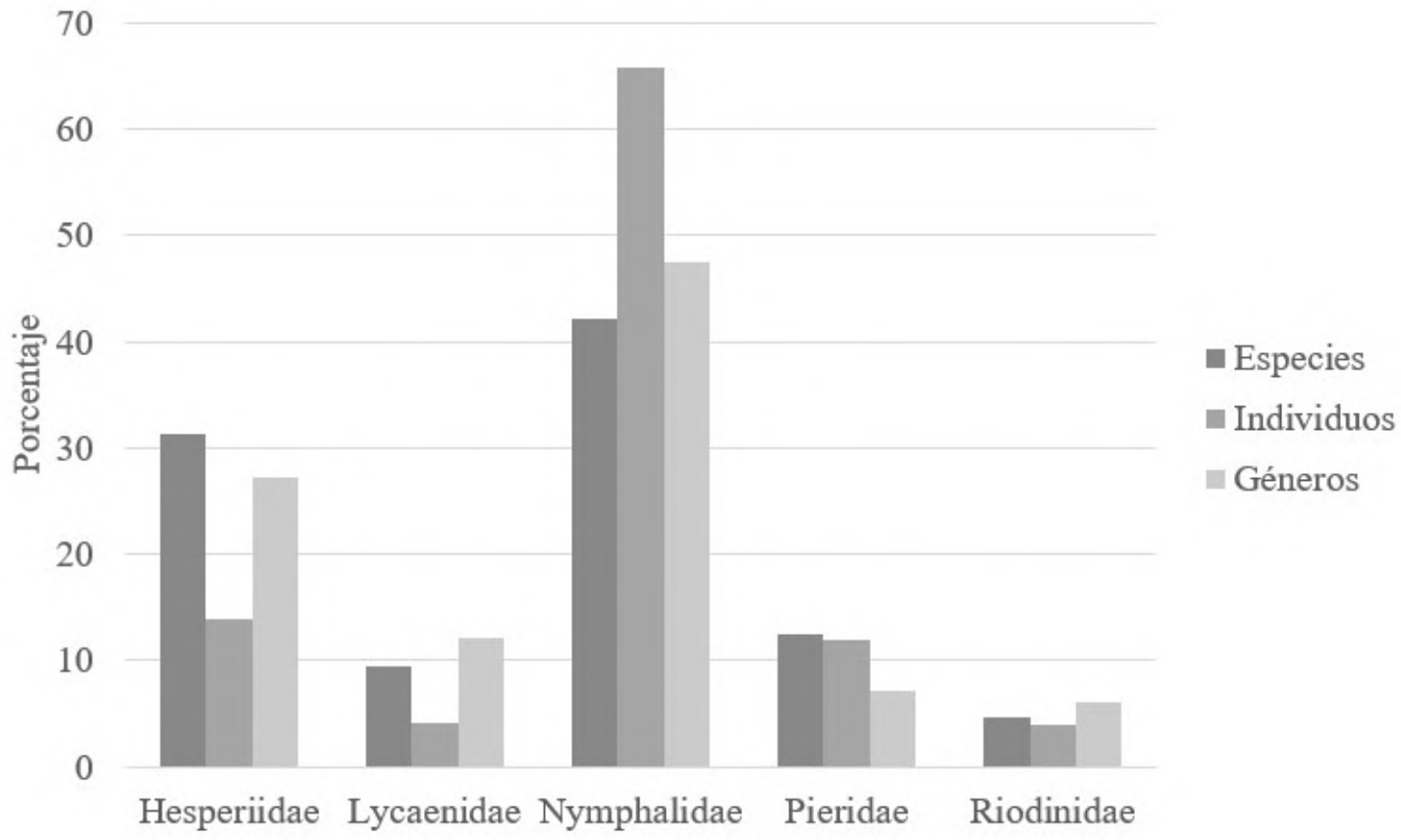

Figura 3. Riqueza y abundancia relativas de familias de papilionoideos en San Andrés de Pisimbalá.

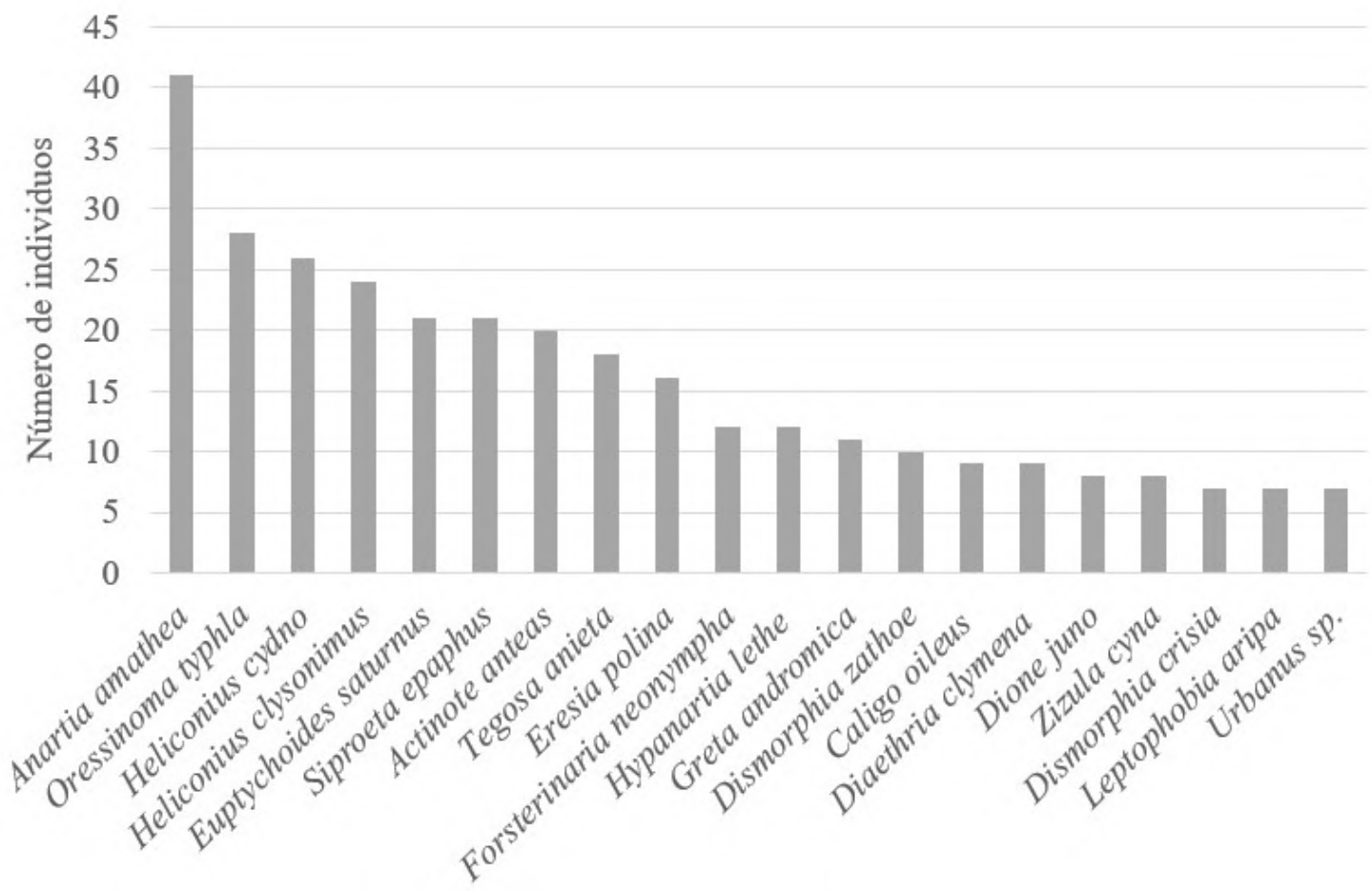

Figura 4. Especies dominantes encontradas en la zona de estudio. 


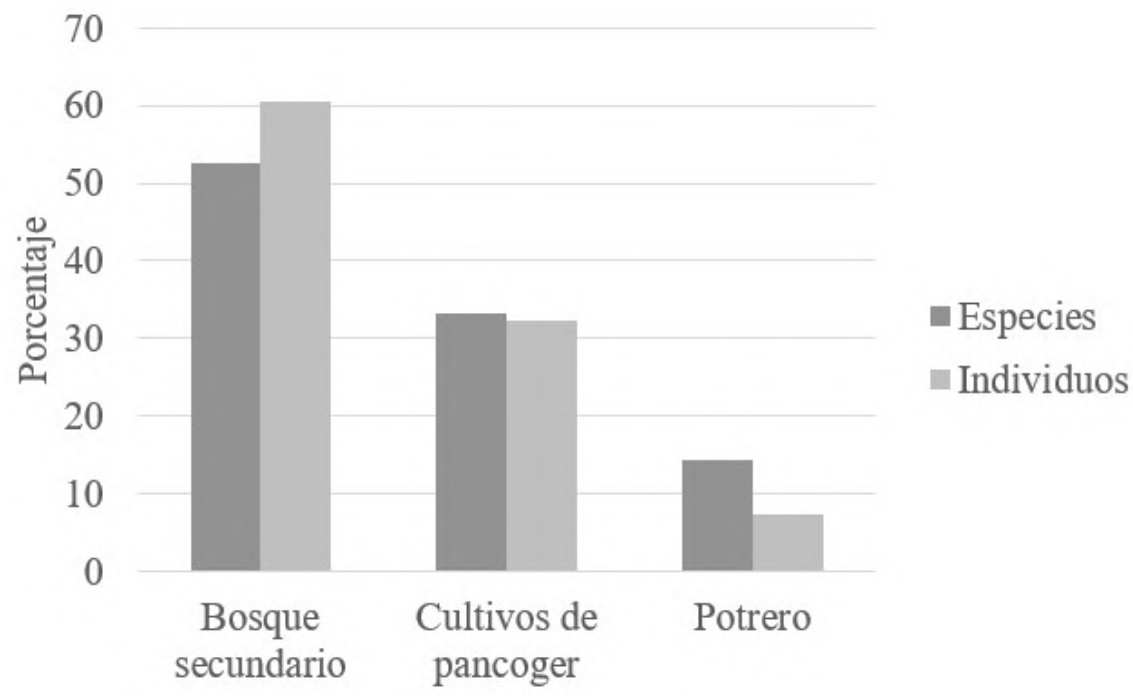

Figura 5. Valores relativos del número de especies e individuos de papilionoideos para cada tipo de cobertura.

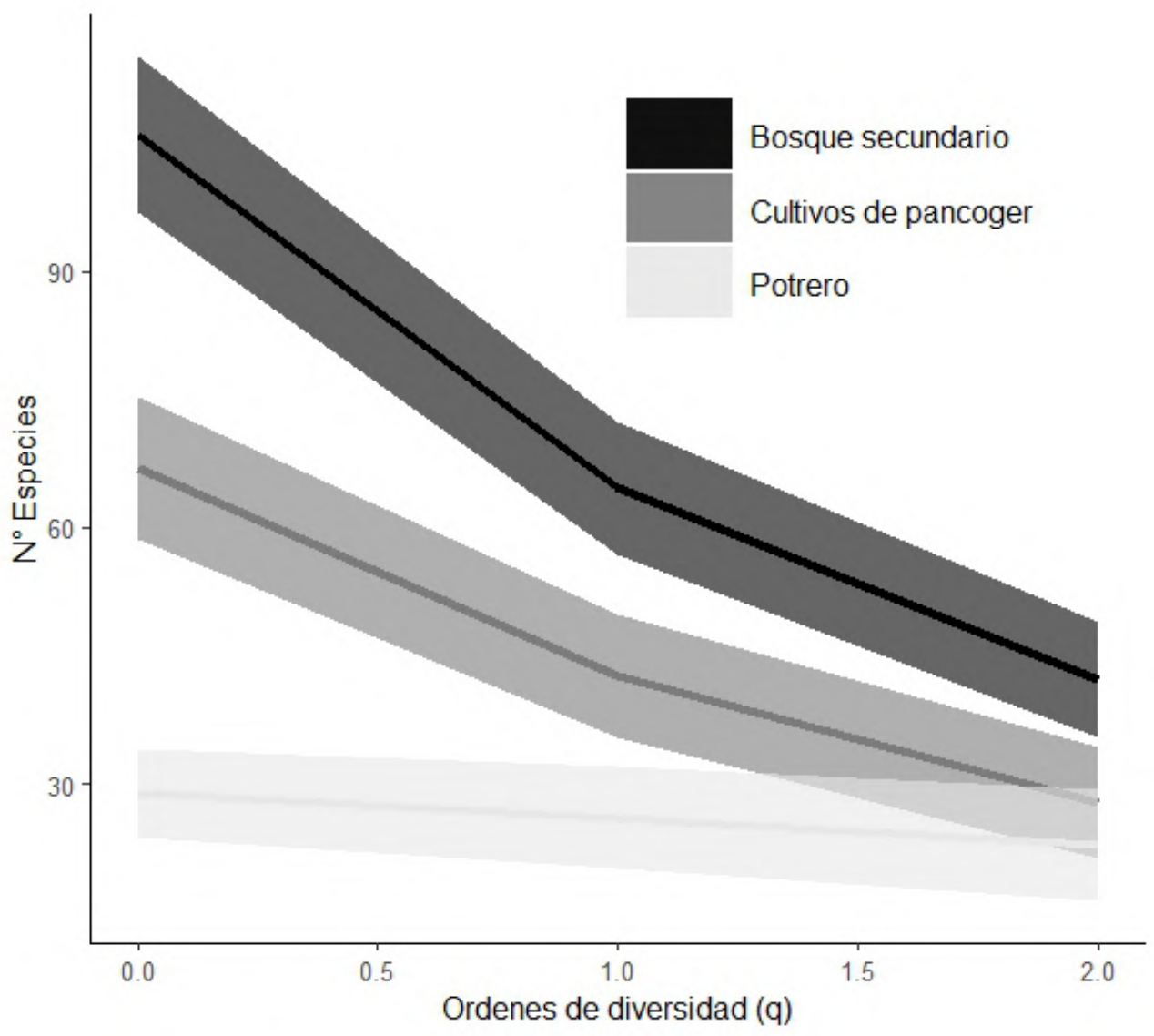

Figura 6. Diversidad verdadera para cada tipo de cobertura. Las áreas sombreadas señalan los intervalos de confianza del $95 \%$ correspondiente a cada tipo de hábitat. 
Tabla 2. Diversidad verdadera para cada tipo de cobertura

\begin{tabular}{l|c|c|c}
\hline Tipo de cobertura & ${ }^{0} \mathbf{q}$ & ${ }^{1} \mathbf{q}$ & ${ }^{2} \mathbf{q}$ \\
\hline Bosque secundario & 106 & 64,63 & 42,28 \\
Cultivos de & 67 & 42,17 & 27,27 \\
pancoger & 29 & 26,04 & 22,85 \\
\hline Potrero &
\end{tabular}

${ }^{0} \mathbf{q}$ : diversidad de orden $0,{ }^{1} \mathbf{q}$ : diversidad de orden 1 , ${ }^{2} \mathbf{q}$ : diversidad de orden 2 .

En San Andrés de Pisimbalá no se han realizado estudios sobre biodiversidad de mariposas, por lo que la riqueza registrada durante la investigación representa el primer registro sobre la caracterización de estos organismos. Sin embargo, se han encontrado mayor número de especies en otros estudios realizados sobre la cordillera central como el de Arias y Huertas (2001) con 144 especies y Ospina et al. (2015) con 266 especies registradas.

La mayor riqueza y abundancia de papilionoideos en la zona de estudio estuvo representada por especies de la familia Nymphalidae; esta familia se encuentra ampliamente distribuida en el país tal como lo repor- tan en sus trabajos Campos y Andrade (2007); Fagua et al. (1999), Orozco et al. (2009) y Prieto y Constantino (1996). Además, Campos y Andrade (2007) enfatizan en el hecho de que dicha familia es muy numerosa en el trópico debido a que se compone de un mayor número de subfamilias, géneros y especies representando el $31 \%$ de mariposas del Neotrópico y el $30 \%$ de especies registradas para Colombia. Sumado a esto, la incidencia de una amplia gama de especies generalistas dentro de esta familia permite explorar diversos recursos alimenticios, mostrando así una ventaja adaptativa frente a otras especies pertenecientes a familias como Riodinidae, Lycaenidae y Hesperiidae donde el registro de recursos no es tan amplio (DeVries, 1987; Ospina y Reinoso, 2009).

La especie más abundante fue $A$. amathea; esta es una especie reportada en toda Colombia, tanto en bosque seco como húmedo hasta los $2000 \mathrm{~m}$ s.n.m (Orozco et al., 2009). Esta distribución permite reconocerla como indicadora de áreas abiertas e intervenidas (Tobar et al., 2002; Valencia et al., 2005). Su abundancia se puede atribuir a su tipo de alimentación en estado adulto, ya que es una mariposa que aprovecha el néctar de flores de plantas ornamentales de familias como Acanthaceae, Asteraceae, Melastomataceae, Rubiaceae, Lamiaceae, Solanaceae y Ver-

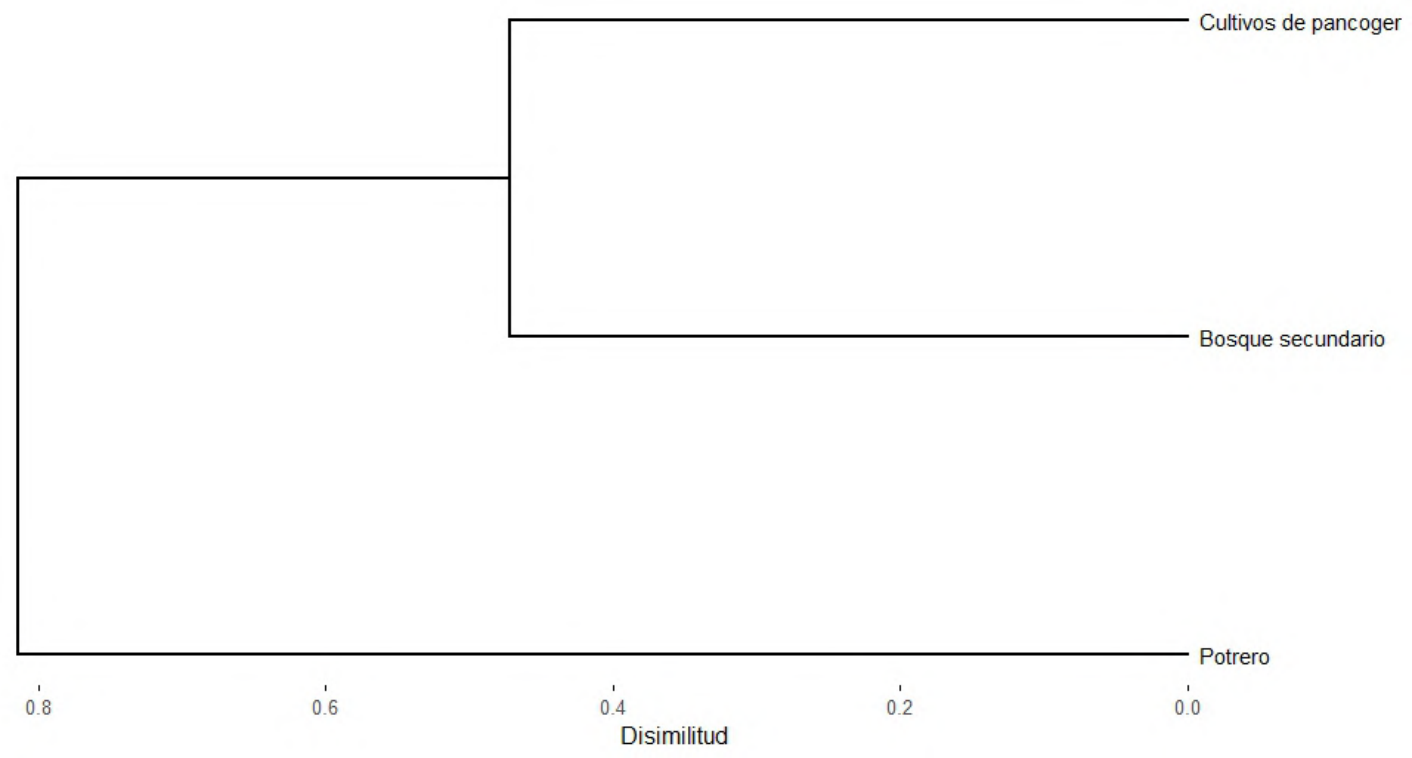

Figura 7. Índice de similitud de Bray-Curtis entre diferentes tipos de cobertura. 
benaceae que fueron registradas en la zona (Andrade, 2002; DeVries, 1987; Orozco et al., 2009).

La estructura vegetal heterogénea de la zona de estudio permite el establecimiento de especies comunes de interiores de bosque, borde de bosque y cafetales bajo sombrío como $H$. cydno, $H$. clysonimus, Colobura dirce (Linnaeus, 1758), Diaethria clymena (Cramer, 1775), Dione juno (Cramer, 1779), Junonia evarete (Cramer, 1779), O. typhla, Oxeoschistus simplex A. Butler, 1868, Leptophobia aripa (Boisduval, 1836), S. epaphus, T. anieta; cuya presencia se asocia con especies de Passifloraceae, Urticaceae, Amaranthaceae, Annonaceae, y Asteraceae (Carrero et al., 2013; DeVries, 1987; Valencia et al., 2005). Además, estas coberturas presentan un alto grado de humedad al estar asociadas a fuentes hídricas cercanas como la quebrada de San Andrés y la Virgen, donde especies como Rhetus dysonii (Saunders, 1850) y Lieinix nemesis (Latreille, [1813]) liban en las orillas arenosas o cerca de ellas (Le Crom et al., 2004). Adicionalmente, es importante destacar el registro de dos especies indicadoras de áreas conservadas como C. oileus (Palacios et al., 2018) y Episcada polita Weymer, 1899 (Andrade et al., 2007), que se encontraron en zonas de sucesión vegetal secundaria tardía, siendo la cobertura con la más alta conectividad biológica observada.

También es de resaltar que en algunas zonas del bosque secundario se encontraron especies indicadoras de áreas abiertas como A. amathea, A. anteas, S. epaphus, E. polina, Altinote stratonice (Latreille, [1813]), Castilia eranites (Hewitson, 1857) y D. juno (Andrade, 2002; Andrade et al., 2007; Carrero et al., 2013; Palacios et al., 2018; Salazar et al., 2010; Tobar et al., 2002). Lo anterior puede deberse a que esta cobertura presenta, en algunos sectores, un mayor nivel de luminosidad por la entresaca selectiva (Prieto y Constantino, 1996), generando cambios en el microclima y las condiciones físicas tales como régimen de luz, temperatura y humedad (Ospina, 2014). Circunstancias que favorecen la colonización de plantas de la familia Asteraceae que es característica de hábitats abiertos, intervenidos y zonas de transición (Casas et al., 2017; Vélez et al., 2015) y frecuentada por mariposas nectarívoras. Algunos caminos de herradura al interior de esta cobertu- ra presentaban materia orgánica en descomposición producto de los desechos de animales como vacas y caballos, al igual que cebos naturales como frutos caídos lo que generaba el registro de una amplia variedad de mariposas generalistas (Prieto y Constantino, 1996) como Zizula cyna (W. H. Edwards, 1881) que adicionalmente visita flores de Lantana camara (Andrade et al., 2007; Salazar et al., 2010) observada en el sitio o Pronophila unifasciata Lathy, 1906 que es considerada generalista y no requiere condiciones ambientales específicas (Coral y Pérez, 2017; Orozco et al., 2009).

En cuanto a los perfiles de diversidad, es importante mencionar que el bosque secundario y los cultivos de pancoger presentan una mayor riqueza de especies efectivas en comparación con el potrero que arrojó el valor más bajo; probablemente, esto se debe a que existe una mayor oferta alimenticia en el bosque secundario y los cultivos de pancoger tanto para especies generalistas como especialistas. Esto contrasta con otros trabajos donde se registra la mayor diversidad en este tipo de coberturas (Coral y Pérez, 2017; Millán et al., 2009). Respecto al orden ${ }^{1} \mathrm{q}$ se destaca a $C$. oileus, $T$. anieta, $H$. clysonimus, $O$. typhla y $S$. epaphus quienes son las especies más frecuentes en bosque y cultivos. Esto posiblemente se deba a las condiciones medio ambientales de estas coberturas que favorecen su tránsito y reproducción (Ríos, 2007). En términos de dominancia (orden ${ }^{2} \mathrm{q}$ ), en las tres coberturas muestreadas se registraron especies dominantes como $A$. amathea y $O$. typhla. De igual manera Rueda y Andrade (2016) encontraron que algunas áreas abiertas como cultivos y potreros tienen una dominancia de mariposas relativamente altas, posiblemente por la amplia abundancia de brinzales y latizales que ofrecen recursos como plantas hospederas y alimenticias.

En la zona de estudio es evidente la diferencia de riqueza y abundancia de papilionoideos entre las coberturas vegetales, debido a que estos parámetros dependen de las condiciones físico-biológicas del hábitat que determinan la tolerancia fisiológica de las mismas, generando dinámicas de depredación y competencia (Simonson et al., 2001). No obstante, se observa una similitud del $52,8 \%$ entre bosque secundario y cultivos de pancoger en cuanto a la 
estructura de mariposas como consecuencia de la interacción entre hábitats adyacentes, ya que ambas coberturas se componen de vegetación heterogénea y representan una gran parte de la zona de estudio. Sumado a esto, la actividad agrícola que se destaca en la zona es la producción de café bajo sombrío, por lo que se pueden hallar cafetales mezclados con otro tipo de vegetación y aledaños a relictos o fragmentos de bosque, que según Coral y Pérez (2017) favorecen una mayor presencia de especies de lepidópteros, generando así una mayor conectividad biológica en la región.

Finalmente, se concluye que las condiciones del área de estudio, a pesar del desarrollo de actividades antrópicas como expansión de zonas agrícolas y pastoriles, aún permiten sustentar una amplia diversidad de especies de papilionoideos que deben ser objeto de estudio y conservación por parte de la comunidad. Se debe destacar que el reconocimiento de la diversidad de estas especies en una zona de interés cultural y ecoturística para el país contribuirá con información de línea base para apoyar propuestas encaminadas hacia un turismo sostenible que se viene dando en la región. También se resalta la importancia del presente estudio como uno de los pioneros sobre el conocimiento de la biodiversidad en Tierradentro, vereda de San Andrés de Pisimbalá.

\section{AGRADECIMIENTOS}

Los autores agradecen, especialmente, al resguardo indígena de San Andrés de Pisimbalá y a su comunidad por el permiso para el desarrollo del presente estudio. También a la familia Velasco Rojas por la acogida en su hogar durante la investigación. Por último, al Grupo de Estudios en Geología, Ecología y Conservación - GECO de la Universidad del Cauca por el préstamo de equipos y el acompañamiento durante el estudio.

\section{CONFLICTO DE INTERESES}

Los autores declaran que no existe ningún conflicto de interés con la investigación presentada.

\section{REFERENCIAS}

Andrade, G. (1990). Clave para las familias y subfamilias de Lepidoptera: Rhopalocera de Colombia. Caldasia, 16 (77), 197-200. https://revistas.unal.edu.co/index. $\mathrm{php} / \mathrm{cal} /$ article/view/35524

Andrade, G. (1995). Nymphalidae, Acraeinae: Actinote. (I. de C. N. (Bogotá). Instituto de Ciencias Naturales-Museo de Historia Natural.

Andrade, G. (1998). Utilización de las mariposas como bioindicadores del tipo de hábitat y su biodiversidad en Colombia. Revista de la Academia Colombiana de Ciencias Exactas, Fúsicas y Naturales, 22(84), 407-421. http: //www.accefyn.com/revista/Vol_22/84/407-421.pdf

Andrade, G. (2002). Biodiversidad de las mariposas (Lepidoptera: Rhopalocera) de Colombia. Red Iberoamericana de Biogeografía y Entomología Sistemática, 2, 153-172. http://sea-entomologia.org/PDF/M3M PRIBES_2002/153_172_Andrade.pdf

Andrade, G., García, E. A., \& García, G. A. (2007). Libro Rojo de los Invertebrados Terrestres de Colombia. Instituto de Ciencias Naturales-Universidad Nacional de Colombia, Conservación Internacional Colombia Instituto Alexander von Humboldt, Ministerio de Ambiente, Vivienda y Crédito Territorial. http://www.bio-nica.info/ Biblioteca/Amat-Garcia2007LibrRojoInvertebrados.pdf

Andrade, G. (2011). Estado del conocimiento de la biodiversidad en Colombia y sus amenazas. Consideraciones para fortalecer la interacción ciencia-política. Revista de la Academia Colombiana de Ciencias Exactas, Físicas y $\mathrm{Na}$ turales, 35(137), 491-508. http://accefyn.org.co/revista/ Vol_35/137/492-508.pdf

Andrade, G., Henao, E., \& Triviño, P. (2013). Ciencias naturales técnicas y procesamiento para la recolección, preservación y montaje de mariposas en estudios de biodiversidad y conservación. (Lepidoptera: Hesperoidea Papilionoidea). Revista de la Academia Colombiana de Ciencias Exactas, Físicas y Naturales, 37(144), 311-325. DOI:10.18257/raccefyn.12

Arias, J. J., \& Huertas, B. (2001). Mariposas diurnas de la Serranía de los Churumbelos, Cauca. Distribución altitudinal y diversidad de especies (Lepidoptera: Rhopalocera: Papilionoidea). Revista Colombiana de Entomología, 27(3-4), 169 - 176.

Campos, L., \& Andrade, G. (2007). Lepidópteros. En: J. O. Rangel (Ed). Estudio de la Caracterización Biológica y Ecológica Integral, Fase I: Diagnóstico, Evaluación y Planificación del Proceso de Recuperación, Protección y Conservación del Bosque Natural del Agüil en el Municipio de Aguachica y Fase II: Formulación del área Protectora del Agüil, Municipio de Aguachica-Cesar. (pp. 103-106). Corporación Autónoma Regional del César y Universidad Nacional de Colombia. https://www.corpocesar.gov.co/ files/Informe\%20Final-Final\%20del\%20Aguil\%202.pdf

Carrero, D., Sánchez, L., \& Tobar, D. (2013). Diversidad y distribución de mariposas diurnas en un gradiente altitudinal en la Región Nororiental Andina de Colombia. Boletín Cientifico Centro de Museos. Museo de Historia Natural, 17(1), 168-188. http://190.15.17.25/ 
boletincientifico/downloads/Boletin(17)1_15.pdf

Casas, L. C., Mahecha, O., Dumar, J. C., \& Ríos, I. C. (2017). Diversidad de mariposas en un paisaje de bosque seco tropical, en la Mesa de los Santos, Santander, Colombia. (Lepidópteros: Papilionoidea). SHILAP Revista de Lepidopterología, 45(177), 83-108. https://www.redalyc.org/ articulo.oa? id $=45550375010$

Clerici, N., Armenteras, D., Karieva, P., Botero, R., Ramírez, J. P., Forero, G., Ochoa, J., Pedraza, C., Schneider, L., Lora, C., Gómez, C., Linares, M., Hirashiki, C., \& Biggs, D. (2020). Deforestation in Colombian protected areas increased during post-conflict periods. Scientific Reports, 10(1), Article 4971. DOI:10.1038/s41598-020-61861-y

Chao, A., \& Jost, L. (2012). Coverage-based rarefaction and extrapolation: standardizing samples by completeness rather than size. Ecology, 93(12), 2533-2547. DOI:10.1890/11-1952.1

Chao, A., \& Jost, L. (2015). Estimating diversity and entropy profiles via discovery rates of new species. Methods in Ecology and Evolution, 6(8), 873-882. DOI:10.1111/2041210X.12349

Chao, A., Ma. K., Hsieh, T., \& Chiu, C. (2015). Online Program SpadeR: Species-richness Prediction and Diversity Estimation in R. Versión 0.1.1. Recuperado de la URL http://chao.stat.nthu.edu.tw/wordpress/software_ download/

Constantino, M. (1995). Revisión de la tribu Haeterini HerrichSchäffer, 1864 en Colombia (Lepidoptera, Nymphalidae: Satyrinae). SHILAP Revista de Lepidopterología, 23(89), 49-76. DOI:10.13140/RG.2.1.2758.5046

Coral, N., \& Pérez, J. (2017). Diversidad de mariposas diurnas (Lepidoptera: Papilionoidea) asociadas a un agroecosistema cafetero de sombra (Curití, Santander). Revista Colombiana de Entomología, 43(1), 91-99. DOI:10.25100/socolen.v43i1.6655

Daily, G., \& Ehrlich P. (1995). Preservation of biodiversity in small rainforest patches: rapid evaluations using butterfly trapping. Biodiversity and Conservation, 4(1), 35-55. DOI:10.1007/BF00115313

D'Abrera, B. (1984). Butterflies of South America. Hill House Publishers.

D'Abrera, B. (1987a). Butterflies of the Neotropical Region. Part III. (Brassolidae, Acraeidae, Nymphalidae, part.). Hill House Publishers.

D'Abrera, B. (1987b). Butterflies of the Neotropical Region. Part IV. Nymphalidae (partim). Hill House Publishers.

D'Abrera, B. (1994). Butterflies of the Neotropical Region. Part VI. Riodinidae. Hill House Publishers.

D'Abrera, B. (1995). Butterflies of the Neotropical Region. Part VII. Lycaenidae. Hill House Publishers.

DeVries, P. (1987). Hostplant records and natural history notes on Costa Rican butterflies (Papilionidae, Pieridae \& Nymphalidae). The Journal of Research on the Lepidoptera, 24 (4), 290-333. https://cmapspublic2.ihmc.us/rid= 1G828QVN0-1T31HBG-12YL/Host_Plant_Notes.pdf

Fagua, G., Amarillo, A., \& Andrade, G. (1999). Mariposas como indicadoras del grado de intervención en la cuenca del río Pato (Caquetá - Colombia). En: G. Amat, G. Andrade, F. Fernández (Eds). Insectos de Colombia (pp. 285-315). Revista de la Academia Colombiana de
Ciencias Exactas, Físicas y Naturales.

García, C., Constantino, L., Heredia, M., \& Kattan, G. (2002). Guía de Campo: Mariposas Comunes de la Cordillera Central de Colombia. Wildlife Conservation Society. DOI:10.13140/RG.2.1.4135.0563

Henao, E. F., \& Gantiva, C. H. (2020). Mariposas (Lepidoptera: Hesperioidea-Papilionoidae) del bosque seco (BST) en Colombia. Conociendo la diversidad en un ecosistema amenazado. Boletín Cientifico. Centro de Museos. Museo de Historia Natural, 24(1), 150-196. DOI:10.17151/bccm.2020.24.1.10

Holdridge, L. (1967). Life zone ecology. Tropical Science Center. http://reddcr.go.cr/sites/default/files/ centro-de-documentacion/holdridge_1966_-_life_zone_ ecology.pdf

Hsieh, T., Ma, K., \& Chao, A. (2013). iNEXT online: interpolation and extrapolation. Versión 1.0. Disponible en http://chao.stat.nthu.edu.tw/blog/software-download/

Jost, L. (2006). Entropy and diversity. Oikos, 113, 363-375. DOI:10.1111/j.2006.0030-1299.14714.x

Lamas, G. (2004). Atlas of Neotropical Lepidoptera. Checklist: Part $4^{a}$ (Hesperioidea-Papilionoidea). Association for Tropical Lepidoptera \& Scientific Publishers.

Le Crom, J., Constantino, L., \& Salazar, J. (2002). Mariposas de Colombia. Tomo I. Papilionidae. Carlec Ltda.

Le Crom, J., Llorente, J., Constantino, L., \& Salazar, J. (2004). Mariposas de Colombia. Tomo II: Pieridae. Carlec Ltda.

Millán, C., Chacón, P., \& Giraldo, A. (2009). Estudio de la comunidad de lepidópteros diurnos en zonas naturales y sistemas reproductivos del municipio de Caloto (Cauca, Colombia). Boletín Científico Centro de Museos. Museo de Historia Natural, 13(1), 185-195.

Ministerio de Ambiente y Desarrollo Sostenible de Colombia (MADS), \& Programa de las Naciones Unidas para el Desarrollo (PNUD). (2014). V Informe nacional de la biodiversidad de Colombia ante el convenio de diversidad biológica. Ministerio de Ambiente y Desarrollo Sostenible. https://www.minambiente.gov.co/images/ sala-de-prensa/Documentos/2014/marzo/310314_v_ informe_bio_colombia_070314.pdf

Morales, N. E. (2011). '?Qué es un bioindicador? Aprendiendo a partir del ciclo de indagación guiada con macroinvertebrados bentónicos, propuesta metodológica [Trabajo de Grado, Universidad Nacional de Colombia]. Repositorio UNAL. https://repositorio.unal.edu.co/handle/unal/ 19984

Oksanen, J., Blanchet, F. G., Friendly, M., Kindt, R., Legendre, P., McGlinn, D., Minchin, P. R., O'Hara, R. B., Simpson, G. L., Solymos, P., Stevens, M. H. H., Szoecs, E., \& Wagner, H. (2020). Vegan: Community Ecology Package. $\mathrm{R}$ package version 2.5-7. https://CRAN.R-project.org/ package $=$ vegan

Orozco, S., Muriel, S., \& Palacio, J. (2009). Diversidad de lepidópteros diurnos en un área de bosque seco tropical del occidente Antioqueño. Actualidades Biológicas, 31 (90), 31-41. https://revistas.udea.edu.co/index.php/ actbio/article/view/4727/4150

Ospina, L. A., \& Reinoso, G. (2009). Mariposas diurnas (Lepidoptera: Papilionoidea y Hesperioidea) del jardín botánico Alejandro von Humboldt de la Univer- 
sidad del Tolima (Ibagué Colombia). Revista Tumbaga, 1(4), 135-148. https://dialnet.unirioja.es/servlet/ articulo? codigo $=3632038$

Ospina, L. A. (2014). Estructura de la comunidad de mariposas diurnas (Lepidoptera: Hesperioidea y Papilionoidae) en distintos tipos de hábitats en la cuenca río Lagunillas (Tolima-Colombia) [Trabajo de Grado, Universidad Nacional de Colombia]. Repositorio UNAL. https: //repositorio.unal.edu.co/handle/unal/53198

Ospina, L. A., Andrade, G., \& Reinoso, G. (2015). Diversidad de mariposas y su relación con el paisaje en la cuenca del río Lagunillas, Tolima, Colombia. Revista de la Academia Colombiana de Ciencias Exactas, Físicas y Naturales, 39(153), 455. DOI:10.18257/raccefyn.215

Pachón, X., Oliveros, D. E., \& Wiesner, L. E. (1996). Geografía humana de Colombia Región Andina Central, Tomo VI, Vol. II. Instituto Colombiano de Cultura Hispánica. https://babel.banrepcultural.org/digital/collection/ p17054coll10/id/2778/

Palacios, V., Palacios, L., \& Jiménez, A. (2018). Diversidad de mariposas diurnas (Lepidoptera: Papilionoidea) asociadas con tres hábitats en el corregimiento de Pacurita, municipio de Quibdó, Chocó, Colombia. Revista de la Academia Colombiana de Ciencias Exactas, Fúsicas y Naturales, 42(164), 237. DOI:10.18257/raccefyn.607

Pollard, E., \& Yates, T. (1996). Monitoring butterflies for ecology and conservation. Springer Netherlands.

Prieto, A., \& Constantino, L. (1996). Abundancia, distribución y diversidad de mariposas en el Río Tatabro, Buenaventura (Valle-Colombia). Boletín Museo Entomológico Universidad del Valle, 4(2), 11-18. https: //bibliotecadigital.univalle.edu.co/bitstream/handle/ 10893/4742/2-\%20Abundancia, \%20distribuci\%F3n\% 20y\%20diversidad $\% 20$ de $\% 20$ mariposas.pdf;jsessionid= 9CF5B321187EB2ED752D94A25696043C?sequence $=1$

R Core Team (2021). R: A language and environment for statistical computing. R Foundation for Statistical Computing, Vienna, Austria. https://www.R-project.org/.

Ríos, C. (2007). Riqueza de especies de Mariposas (Hesperioidea \& Papilionoidea) de la quebrada "El Aguila" Cordillera Central (Manizales - Colombia). Boletín Científico Centro de Museos. Museo de Historia Natural, 11(1), 272-291. http://www.scielo.org.co/pdf/bccm/ v11n1/v11n1a13.pdf

Rueda, N., \& Andrade, M. (2016). El género Heliconius Kluk, 1708 en dos hábitats de diferente grado de conservación en la Amazonia colombiana y aportes para su conservación. Revista de la Academia Colombiana de Ciencias Exactas, Físicas y Naturales, 40(157), 653-663. DOI:10.18257/raccefyn.382

Salazar, J., Vargas, J., Mora, A., \& Benavides, J. (2010). Identificación Preliminar de los Rhopalocera que Habitan el Centro Experimental Amazónico (C.E.A.) Mocoa-Putumayo y Algunas Especies Aptas Para Criar en Cautiverio (Insecta: Lepidoptera). Boletín Científico Centro de Museos. Museo de Historia Natural, 14(1), 150-188. http://190.15.17.25/boletincientifico/ downloads/Boletin14(1)_11.pdf

Sánchez, G. (2002). Desarrollo y medio ambiente: una mirada a Colombia. Economía y Desarrollo, 1(1), 79-98. http:
//uac1.fuac.edu.co/revista/M/seis.pdf

Simonson, S., Opler, P., Stohlgren, T., \& Chong, G. (2001). Rapid assessment of butterfly diversity in a montane landscape. Biodiversity and Conservation, 103(3), 239248. DOI:10.1023/A:1016663931882

Tacuma, D. (2020). Los impactos ambientales de los cultivos ilícitos en las áreas naturales protegidas en Colombia: problemáticas y soluciones [Trabajo de grado, Universidad Católica de Colombia]. Repositorio Ucatólica. https://repository.ucatolica.edu.co/handle/10983/24916

Tobar, L., Rangel, J., \& Andrade, G. (2002). Diversidad de mariposas (Lepidoptera: Rhopalocera) en la parte alta de la cuenca del río El Roble (Quindío-Colombia). Caldasia, 24 (2), 393-409. https://revistas.unal.edu.co/index.php/ $\mathrm{cal} /$ article/view/39422

Valencia, M., Gil, C., \& Constantino, Z. (2005). Mariposas diurnas de la zona central cafetera colombiana. Guía de campo. Cenicafé. https://biblioteca.cenicafe.org/handle/ 10778/639

Vélez, D., Gallego, M., \& Riascos, Y. (2015). Diversidad De Mariposas Diurnas (Insecta: Lepidóptera) de un Bosque Subandino, Cajibío, Cauca. Boletín Científico Centro de Museos. Museo de Historia Natural, 19(1), 263-285. DOI:10.17151/bccm.2015.19.1.20

Villalobos, A. \& Salazar, J. A. (2020). Mariposas (Lepidoptera: Papilionoidea) de un bosque Andino en la vertiente oriental de la cuenca de río Tona, Santander (Colombia). Anales de Biología, 42(168), 75-84. https://revistas.um. es/analesbio/article/view/429621

Villarreal, H., Álvarez, M., Córdoba, S., Escobar, F., Fagua, G., Gast, F., \& Umaña, A. (2006). Manual de métodos para el desarrollo de inventarios de biodiversidad. Programa de inventarios de biodiversidad. Instituto de Investigación de Recursos Biológicos Alexander von Humboldt. http://repository.humboldt.org.co/bitstream/handle/ 20.500.11761/31419/63.pdf?sequence $=1$

Warren, A. D., Davis, K. J., Stangeland, E. M., Pelham, J. P., Willmott, K. R., \& Grishin, N. V. (2017). Illustrated Lists of American Butterflies. https://www. butterfliesofamerica.com/

Wickham, H. (2016). ggplot2: Gráficos elegantes para el análisis de datos. Versión 3.3.2. http://ggplot2.org

Xie, Y. (2014). knitr: A Comprehensive Tool for Reproducible Research in R. En V. Stodden, F. Leisch, \& R. D. Peng (Eds.). Implementing Reproducible Computational Research (pp. 30). Chapman and Hall/CRC. http: //www.crcpress.com/product/isbn/9781466561595. 


\section{Anexos}

Anexo 1.Listado de papilionoideos registrados en San Andrés de Pisimbalá, Cauca, Colombia. P - Potrero, BS - Bosque Secundario, CP - Cultivos de Pancoger

\begin{tabular}{|c|c|c|c|c|}
\hline Especie & & $\mathbf{P}$ & BS & $\mathrm{CP}$ \\
\hline Nymphalidae & & & & \\
\hline & Danaus plexippus (Linnaeus, 1758) & $\mathrm{X}$ & $\mathrm{X}$ & \\
\hline & Thyridia psidii (Linnaeus, 1758) & $\mathrm{X}$ & $\mathrm{X}$ & $\mathrm{X}$ \\
\hline & Mechanitis menapis Hewitson, [1856] & & & $\mathrm{X}$ \\
\hline & Mechanitis sp. Fabricius, 1807 & & $\mathrm{X}$ & $\mathrm{X}$ \\
\hline Danainae & Pagyris cymothoe (Hewitson, [1855]) & & $\mathrm{X}$ & $\mathrm{X}$ \\
\hline & Episcada polita Weymer, 1899 & & $\mathrm{X}$ & $\mathrm{X}$ \\
\hline & Hypoleria lavinia (Hewitson, [1855]) & & $\mathrm{X}$ & \\
\hline & Greta andromica (Hewitson, [1855]) & $\mathrm{X}$ & $\mathrm{X}$ & $\mathrm{X}$ \\
\hline & Greta dercetis (E. Doubleday, 1847) & & & $\mathrm{X}$ \\
\hline & Altinote stratonice (Latreille, [1813]) & $\mathrm{X}$ & $\mathrm{X}$ & $\mathrm{X}$ \\
\hline & Actinote anteas (E. Doubleday, [1847]) & $\mathrm{X}$ & $\mathrm{X}$ & $\mathrm{X}$ \\
\hline & Dione juno (Cramer, 1779) & & $\mathrm{x}$ & $\mathrm{X}$ \\
\hline & Dryas iulia (Fabricius, 1775) & & $\mathrm{X}$ & $\mathrm{X}$ \\
\hline Heliconiinae & Eueides isabella (Stoll, 1781) & & $\mathrm{X}$ & \\
\hline & Heliconius clysonymus Latreille, [1817] & $\mathrm{X}$ & $\mathrm{X}$ & $\mathrm{X}$ \\
\hline & Heliconius cydno (E. Doubleday, 1847) & $\mathrm{X}$ & $\mathrm{X}$ & $\mathrm{X}$ \\
\hline & Heliconius doris (Linnaeus, 1771) & & $\mathrm{X}$ & \\
\hline & Heliconius erato (Linnaeus, 1758) & & $\mathrm{X}$ & \\
\hline Limenitidinae & Adelpha seriphia (C. Felder \& R. Felder, 1867) & & $\mathrm{X}$ & \\
\hline & Biblis hyperia (Cramer, 1779) & & $\mathrm{X}$ & \\
\hline & Cybdelis mnasylus E. Doubleday, [1848] & & & $\mathrm{X}$ \\
\hline Biblidinae & Dynamine aerata (A. Butler, 1877) & & $\mathrm{X}$ & \\
\hline & Dynamine artemisia (Fabricius, 1793) & & $\mathrm{X}$ & \\
\hline & Diaethria clymena (Cramer, 1775) & & $\mathrm{X}$ & $\mathrm{X}$ \\
\hline Cyrestinae & Marpesia zerynthia Hübner, [1823] & & $\mathrm{X}$ & \\
\hline & Pycina zamba E. Doubleday, [1849] & & & $\mathrm{X}$ \\
\hline & Colobura dirce (Linnaeus, 1758) & & $\mathrm{X}$ & $\mathrm{X}$ \\
\hline & Hypanartia lethe (Fabricius, 1793) & & $\mathrm{X}$ & $\mathrm{X}$ \\
\hline & Vanessa virginiensis (Drury, 1773) & $\mathrm{X}$ & $\mathrm{X}$ & $X$ \\
\hline & Anartia amathea (Linnaeus, 1758) & $x$ & $\mathrm{x}$ & $\mathrm{X}$ \\
\hline & Siproeta epaphus (Latreille, [1813]) & $\mathrm{X}$ & $\mathrm{X}$ & $\mathrm{X}$ \\
\hline Nymphalınae & Junonia evarete (Cramer, 1779) & & $\mathrm{X}$ & $\mathrm{X}$ \\
\hline & Chlosyne lacinia (Geyer, 1837) & & $\mathrm{X}$ & $\mathrm{X}$ \\
\hline & Anthanassa drusilla (C. Felder \& R. Felder, 1861) & & $\mathrm{X}$ & \\
\hline & Castilia eranites (Hewitson, 1857) & $\mathrm{X}$ & $\mathrm{X}$ & $\mathrm{X}$ \\
\hline & Eresia polina Hewitson, 1852 & & $\mathrm{X}$ & $\mathrm{X}$ \\
\hline & Tegosa anieta (Hewitson, 1864) & $\mathrm{X}$ & $\mathrm{X}$ & $\mathrm{X}$ \\
\hline & Caligo oileus C. Felder \& R. Felder, 1861 & & $\mathrm{X}$ & $\mathrm{X}$ \\
\hline & Eryphanis sp. Boisduval, 1870 & & $\mathrm{X}$ & \\
\hline & Opsiphanes tamarindi C. Felder \& R. Felder, 1861 & & $\mathrm{X}$ & \\
\hline & Manataria hercyna (Hübner, [1821]) & $\mathrm{X}$ & $\mathrm{X}$ & \\
\hline & Pronophila unifasciata Lathy, 1906 & & & $\mathrm{X}$ \\
\hline & Oxeoschistus simplex A. Butler, 1868 & $\mathrm{X}$ & $\mathrm{X}$ & $X$ \\
\hline Satyrinae & Cissia pompilia (C. Felder \& R. Felder, 1867) & & $\mathrm{x}$ & \\
\hline & Euptychia sp. Hübner, 1818 & & $\mathrm{X}$ & \\
\hline & Euptychoides saturnus (A. Butler, 1867) & $\mathrm{X}$ & $\mathrm{X}$ & $\mathrm{X}$ \\
\hline & Hermeuptychia harmonia (A. Butler, 1867) & & $\mathrm{X}$ & \\
\hline & Hermeuptychia sp. Forster, 1964 & & $\mathrm{X}$ & \\
\hline & Magneuptychia sp. Forster, 1964 & & $\mathrm{X}$ & \\
\hline
\end{tabular}




\begin{tabular}{|c|c|c|c|c|}
\hline & Pareuptychia metaleuca (Boisduval, 1870) & & $\mathrm{X}$ & \\
\hline & Splendeuptychia ashna (Hewitson, 1869) & & $\mathrm{x}$ & $\mathrm{x}$ \\
\hline & Forsterinaria neonympha (C. Felder \& R. Felder, 1867) & & $\mathrm{x}$ & $\mathrm{X}$ \\
\hline & Taygetis chrysogone E. Doubleday, [1849] & & & $\mathrm{X}$ \\
\hline & Oressinoma typhla E. Doubleday, [1849] & & $\mathrm{X}$ & $\mathrm{X}$ \\
\hline \multicolumn{5}{|l|}{ Hesperiidae } \\
\hline \multirow{10}{*}{ Eudaminae } & Urbanus dorantes (Stoll, 1790) & $\mathrm{X}$ & $\mathrm{X}$ & \\
\hline & Urbanus procne (Plötz, 1881) & $\mathrm{X}$ & & \\
\hline & Urbanus proteus (Linnaeus, 1758) & & $\mathrm{X}$ & \\
\hline & Urbanus sp. Hübner, [1807] & & $\mathrm{X}$ & $\mathrm{X}$ \\
\hline & Urbanus teleus (Hübner, 1821) & $\mathrm{X}$ & $\mathrm{X}$ & $\mathrm{X}$ \\
\hline & Astraptes anaphus (Cramer, 1777) & $\mathrm{X}$ & & \\
\hline & Astraptes fulgerator (Walch, 1775) & & $\mathrm{X}$ & \\
\hline & Astraptes sp. Hübner, [1819] & & $\mathrm{X}$ & \\
\hline & Autochton cf. itylus Hübner, 1823 & $\mathrm{X}$ & $\mathrm{X}$ & $\mathrm{X}$ \\
\hline & Autochton longipennis (Plötz, 1882) & & $\mathrm{X}$ & $\mathrm{X}$ \\
\hline \multirow{14}{*}{ Pyrginae } & Nisoniades cf. suprapanama Steinhauser, 1989 & & $\mathrm{X}$ & \\
\hline & Nisoniades sp. Hübner, [1819] & & & $\mathrm{x}$ \\
\hline & Noctuana lactífera (A. Butler \& H. Druce, 1872) & & $\mathrm{X}$ & \\
\hline & Gorgythion begga (Prittwitz, 1868) & & & $\mathrm{X}$ \\
\hline & Ebrietas sp. Godman \& Salvin, 1869 & & $\mathrm{X}$ & \\
\hline & Theagenes albiplaga (C. Felder \& R. Felder, 1867) & & $\mathrm{X}$ & \\
\hline & Achlyodes pallida (R. Felder, 1869) & & $\mathrm{X}$ & \\
\hline & Quadrus cerialis (Stoll, 1782) & & & $\mathrm{x}$ \\
\hline & Quadrus truncata (Hewitson, 1870) & $\mathrm{X}$ & $\mathrm{X}$ & $\mathrm{X}$ \\
\hline & Xenophanes tryxus (Stoll, 1780) & & & $\mathrm{x}$ \\
\hline & Pyrgus orcus (Stoll, 1780) & $\mathrm{X}$ & $\mathrm{X}$ & \\
\hline & Heliopetes alana (Reakirt, 1868) & & $\mathrm{X}$ & $\mathrm{x}$ \\
\hline & Heliopetes arsalte (Linneaus, 1758) & & $\mathrm{X}$ & \\
\hline & Heliopetes laviana (Hewitson, 1868) & & $\mathrm{x}$ & $\mathrm{x}$ \\
\hline \multirow{16}{*}{ Hesperiinae } & Perichares sp. Scudder, 1872 & & & $\mathrm{X}$ \\
\hline & Zenis jebus (Plötz, 1882) & & $\mathrm{X}$ & \\
\hline & Anthoptus epictetus (Fabricius, 1793) & $\mathrm{X}$ & $\mathrm{X}$ & \\
\hline & Corticea sp. Evans, 1955 & & $\mathrm{x}$ & $\mathrm{x}$ \\
\hline & Mnaseas macia Evans, 1955 & & $\mathrm{X}$ & \\
\hline & Apaustus gracilis (C. Felder \& R. Felder, 1867) & & $\mathrm{x}$ & \\
\hline & Papias phainis Godman, 1900 & $\mathrm{X}$ & & \\
\hline & Tigasis colomus (E. Bell, 1941) & & $\mathrm{X}$ & $\mathrm{X}$ \\
\hline & Vettius coryna (Hewitson, 1866) & & $\mathrm{X}$ & $\mathrm{X}$ \\
\hline & Enosis immaculata (Hewitson, 1868) & $\mathrm{X}$ & $\mathrm{X}$ & \\
\hline & Saturnus reticulata (Plötz, 1893) & & $\mathrm{X}$ & \\
\hline & Pompeius pompeius (Latreille, [1824]) & & $\mathrm{X}$ & \\
\hline & Racta cf. plasma Evans, 1955 & & $\mathrm{X}$ & \\
\hline & Hesperiidae sp1. Latreille, 1809 & & $\mathrm{X}$ & \\
\hline & Hesperiidae sp2. Latreille, 1809 & & & $\mathrm{X}$ \\
\hline & Hesperiidae sp3. Latreille, 1809 & $\mathrm{X}$ & & \\
\hline \multicolumn{5}{|l|}{ Pieridae } \\
\hline \multirow{4}{*}{ Dismorphiinae } & Pseudopieris viridula (C. Felder \& R. Felder, 1861) & & $\mathrm{X}$ & $\mathrm{X}$ \\
\hline & Lieinix nemesis (Latreille, [1813]) & & $\mathrm{X}$ & $\mathrm{X}$ \\
\hline & Dismorphia crisia (Drury, 1782) & & $\mathrm{X}$ & $\mathrm{X}$ \\
\hline & Dismorphia zathoe (Hewitson, [1858]) & & $\mathrm{X}$ & $\mathrm{X}$ \\
\hline \multirow{6}{*}{ Coliadinae } & Eurema albula (Cramer, 1775) & $\mathrm{X}$ & $\mathrm{X}$ & \\
\hline & Eurema salome (C. Felder \& R. Felder, 1861) & & $\mathrm{X}$ & $\mathrm{x}$ \\
\hline & Eurema sp. Hübner, [1819] & & $\mathrm{X}$ & \\
\hline & Eurema xantochlora (Kollar, 1850) & $\mathrm{X}$ & & $x$ \\
\hline & Phoebis neocypris (Hübner, [1823]) & & $\mathrm{X}$ & \\
\hline & Phoebis philea (Linnaeus, 1763) & & $\mathrm{X}$ & \\
\hline
\end{tabular}




\begin{tabular}{|c|c|c|c|c|}
\hline & Phoebis sennae (Linnaeus, 1758) & & $\mathrm{X}$ & $\bar{X}$ \\
\hline & Phoebis sp. Hübner, [1819] & & $\mathrm{X}$ & \\
\hline \multirow{4}{*}{ Pierinae } & Leptophobia aripa (Boisduval, 1836) & & $\mathrm{X}$ & $\mathrm{X}$ \\
\hline & Leptophobia eleone (E. Doubleday, 1847) & & & $x$ \\
\hline & Leptophobia penthica (Kollar, 1850) & & $\mathrm{X}$ & \\
\hline & Catasticta flisa (Herrich-Schäffer, [1858]) & & $\mathrm{X}$ & \\
\hline \multicolumn{5}{|l|}{ Lycaenidae } \\
\hline \multirow{7}{*}{ Theclinea } & Theritas sp. Hübner, 1818 & & $\mathrm{X}$ & \\
\hline & Micandra sp. Staundinger, 1888 & & & $\mathrm{X}$ \\
\hline & Rekoa meton (Cramer, 1779) & & $\mathrm{X}$ & \\
\hline & Arawacus leucogyna (C. Felder \& R. Felder, 1865) & & $\mathrm{X}$ & \\
\hline & Contrafacia imma (Prittwitz, 1865) & & $\mathrm{X}$ & \\
\hline & Arzecla cf. sethon (Godman \& Salvin, 1887) & & $\mathrm{X}$ & \\
\hline & Strymon mulucha (Hewitson, 1867) & & & $\mathrm{X}$ \\
\hline \multirow{5}{*}{ Polyommatinae } & Tmolus echion (Linnaeus, 1767) & & $\mathrm{X}$ & \\
\hline & Panthiades bathildis (C. Felder \& R. Felder, 1865) & & $X$ & \\
\hline & Zizula cyna (W. H. Edwards, 1881) & & $\mathrm{X}$ & $\mathrm{x}$ \\
\hline & Leptotes cassius (Cramer, 1775) & & & $x$ \\
\hline & Cupido cf. comyntas (Godart, [1824]) & $\mathrm{X}$ & $\mathrm{X}$ & \\
\hline \multicolumn{5}{|l|}{ Riodinidae } \\
\hline \multirow{2}{*}{ Euselasiinae } & Euselasia eupatra Seitz, 1916 & & $\mathrm{X}$ & $\mathrm{X}$ \\
\hline & Hades noctula Westwood, 1851 & & $\mathrm{X}$ & $x$ \\
\hline \multirow{4}{*}{ Riodininae } & Mesosemia mevania Hewitson, [1857] & & $\mathrm{X}$ & \\
\hline & Rhetus dysonii (Saunders, 1850) & & $\mathrm{x}$ & \\
\hline & Crocozona pheretima C. Felder \& R. Felder, 1865 & & $\mathrm{X}$ & $\mathrm{X}$ \\
\hline & Eurybia cf. molochina Stichel, 1910 & & & $\mathrm{x}$ \\
\hline
\end{tabular}

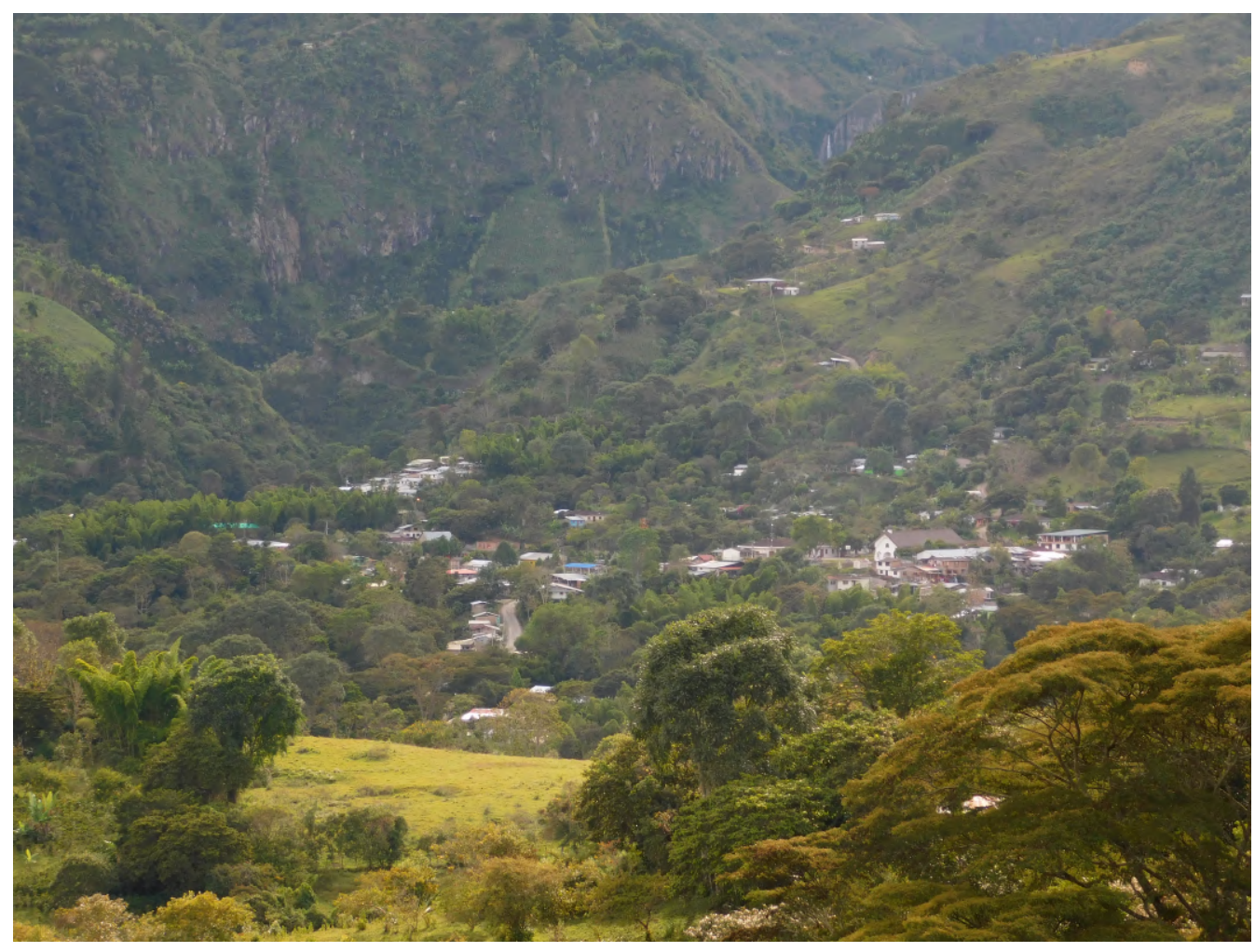

Anexo. 2. Área de estudio, San Andrés de Pisimbalá, Inzá, Cauca. 
A

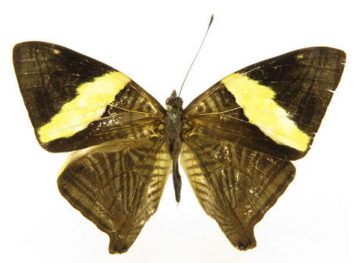

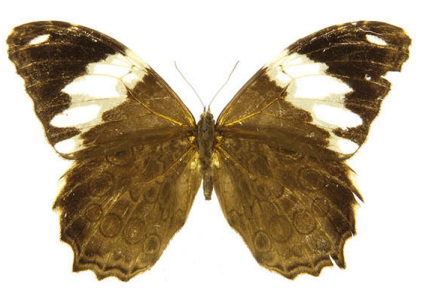

$\mathrm{C}$

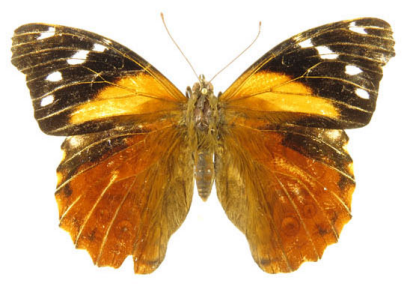

$\mathrm{D}$

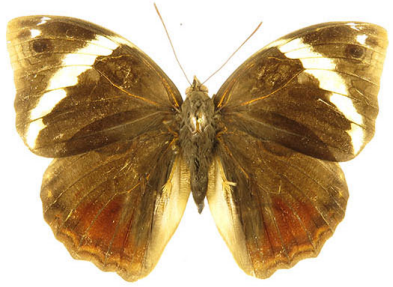

DORSAL
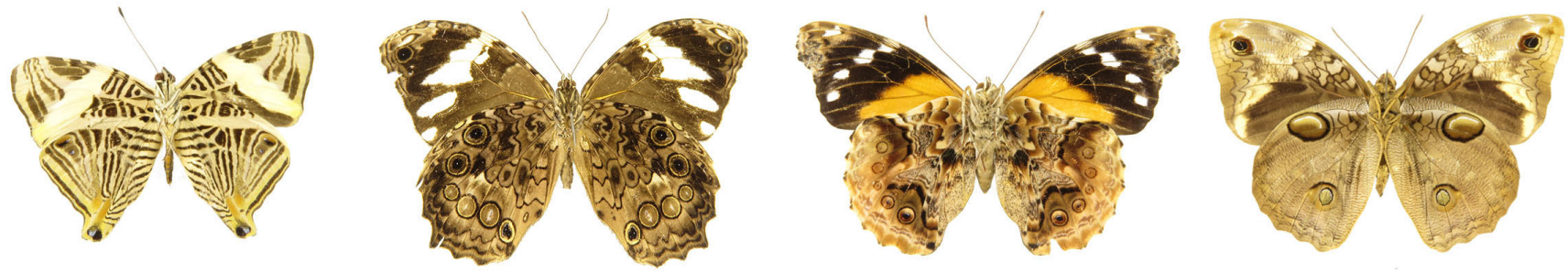

VENTRAL

Anexo. 3. Fotografías de la vista dorsal y ventral de algunas especies registradas en este estudio A. C. dirce; B. M. hercyna; C. P. zamba; D. O. tamarindi. 